\title{
Retroperitoneal Erdheim-Chester disease without skeletal bone involvement mimicking uterine sarcoma with multiple organ involvement
}

\author{
Hae Min Kim, MD ${ }^{1,2}$, Gun Oh Chong, MD, PhD ${ }^{1,2}$, Min Ju Kim, MD ${ }^{1,2}$, Ji Young Park, MD, PhD 3 , \\ Yoon Hee Lee, MD ${ }^{1,2}$ \\ Department of Obstetrics and Gynecology, ${ }^{1}$ School of Medicine, Kyungpook National University, ${ }^{2}$ Kyungpook National University Chilgok Hospital; \\ ${ }^{3}$ Department of Pathology, School of Medicine, Kyungpook National University, Daegu, Korea
}

Erdheim-Chester disease (ECD) is a rare type of non-Langerhans cell histiocytosis and is characterized by the diffuse histiocytic infiltration of multiple organs. Retroperitoneal ECD, especially with uterine involvement, is extremely rare. We report about a 73-year-old woman who presented with vaginal spotting for a month and experienced abdominal pain along with pus-like urine. Computed tomography revealed an irregular mass-like lesion in the uterus, possibly a uterine sarcoma, invading the ureter, rectosigmoid, and bladder. A tissue biopsy of the retroperitoneal mass revealed typical morphological and immunohistochemical features of ECD. However, clinical features, especially long bone involvement, did not coincide with ECD, and BRAF V600E gene mutation was not detected. We made a diagnosis of atypical retroperitoneal ECD mimicking uterine sarcoma with multiple organ involvement.

Keywords: Erdheim-Chester disease; Uterus; Ureter

\section{Introduction}

Histiocytosis is a complex and heterogeneous group of disorders of unknown etiology. Moreover, differentiating the types of histiocytosis can be quite challenging and requires a combination of clinical, radiological, and immunohistochemical features [1]. Erdheim-Chester disease (ECD) is characterized by the accumulation of foamy macrophages, chronic inflammation, fibrosis, and organ failure [2]. ECD tumors contain lipid-laden macrophages that are CD68-positive and CD1a-/ S100-negative [3]. ECD primarily affects the bones and may involve virtually every organ and tissue with protean clinical manifestations, ranging from asymptomatic bone involvement to multisystemic, life-threatening forms [2].

We describe a unique case of multi-retroperitoneal organ involvement, mainly the ureter, kidney, rectum, bladder, and uterus, with no skeletal bone involvement.

\section{Case report}

A 73-year-old woman visited our hospital with vaginal spot- ting for a month. She had also been experiencing abdominal pain along with pus-like urine. Transvaginal ultrasonography revealed a $5-\mathrm{cm}$ mass on the anterior uterus. The mass was suspected to have invaded the bladder and rectum. Laboratory studies revealed an elevated white blood cell count of $12.510^{3} / \mu \mathrm{L}$ and a C-reactive protein level of $23.17 \mathrm{mg} /$ dL. However, other tumor markers (CA125, CA19-9, carcinoembryonic antigen) were within the normal range. Magnetic resonance imaging also revealed a $5.2 \times 5.8 \times 6.8-\mathrm{cm}^{3}$

Received: 2019.12.09. Accepted: 2020.02.27

Corresponding author: Gun Oh Chong, MD, PhD

Department of Obstetrics and Gynecology, Kyungpook National University Chilgok Hospital, 807 Hoguk-ro, Buk-gu, Daegu 41404, Korea

E-mail: gochong@knu.ac.kr

https://orcid.org/0000-0003-4887-4017

Articles published in Obstet Gynecol Sci are open-access, distributed under the terms of the Creative Commons Attribution Non-Commercial License (http://creativecommons org/licenses/by-nc/3.0/) which permits unrestricted non-commercial use, distribution, and reproduction in any medium, provided the original work is properly cited.

Copyright $\odot 2020$ Korean Society of Obstetrics and Gynecology 


\section{Obstetrics \& Gynecology Science}

Hae Min Kim, et al. Retroperitoneal histiocytosis

irregular mass lesion in the uterus that invaded the right pelvic sidewall, bladder, rectosigmoid colon, and right ureter (Fig. 1A and B).

A computed tomography urography scan of her abdomen also revealed a lobulated soft tissue mass in the uterus that invaded the right mid ureter, severe hydronephrosis, and diffuse wall thickening at the rectosigmoid junction of the colon (Fig. 1C).

Explorative laparotomy was performed based on the suspicion of a uterine sarcoma that invaded the right ureter, rectum, and bladder. A lobulated uterine mass approximately
$7 \mathrm{~cm}$ in size was agglomerated with the rectum and bladder and invaded the right ureter and pelvic wall. The right ureter was filled with pus-like fluid. Total abdominal hysterectomy, bilateral salpingo-oophorectomy, low anterior resection and small bowel resection, appendectomy, right nephrectomy, and partial cystectomy were performed. Frozen sections of the uterus, kidney, and rectum showed diffuse histiocytic infiltration. The final histopathology showed diffuse histiocytic infiltration within the entire myometrium, rectum, right ureter, right kidney, and bladder. Tumor cells had large amounts of cytoplasm with a foamy appearance; however, there were
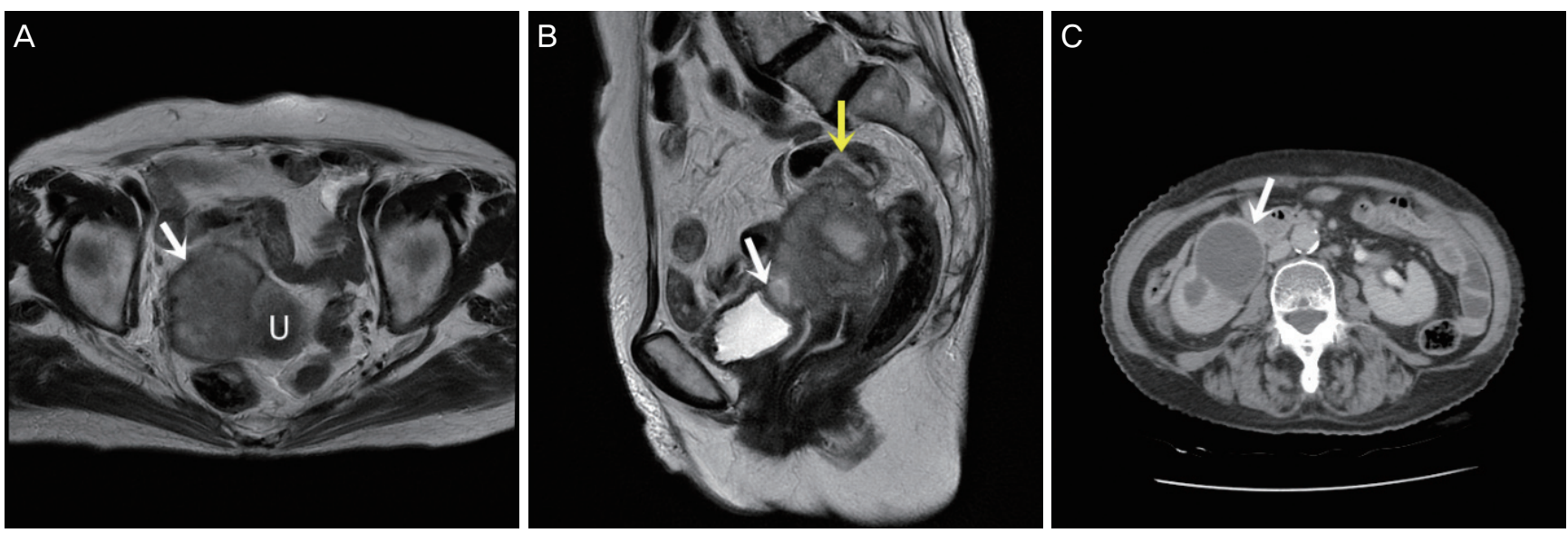

Fig. 1. (A) Magnetic resonance image showing a lobulated irregular mass (white arrow) at the left side of the uterus (U: uterus). (B) Magnetic resonance image showing bladder invasion (white arrow) and rectosigmoid invasion (yellow arrow). (C) Computed tomographic image showing severe hydronephrosis and hydroureter (white arrow).
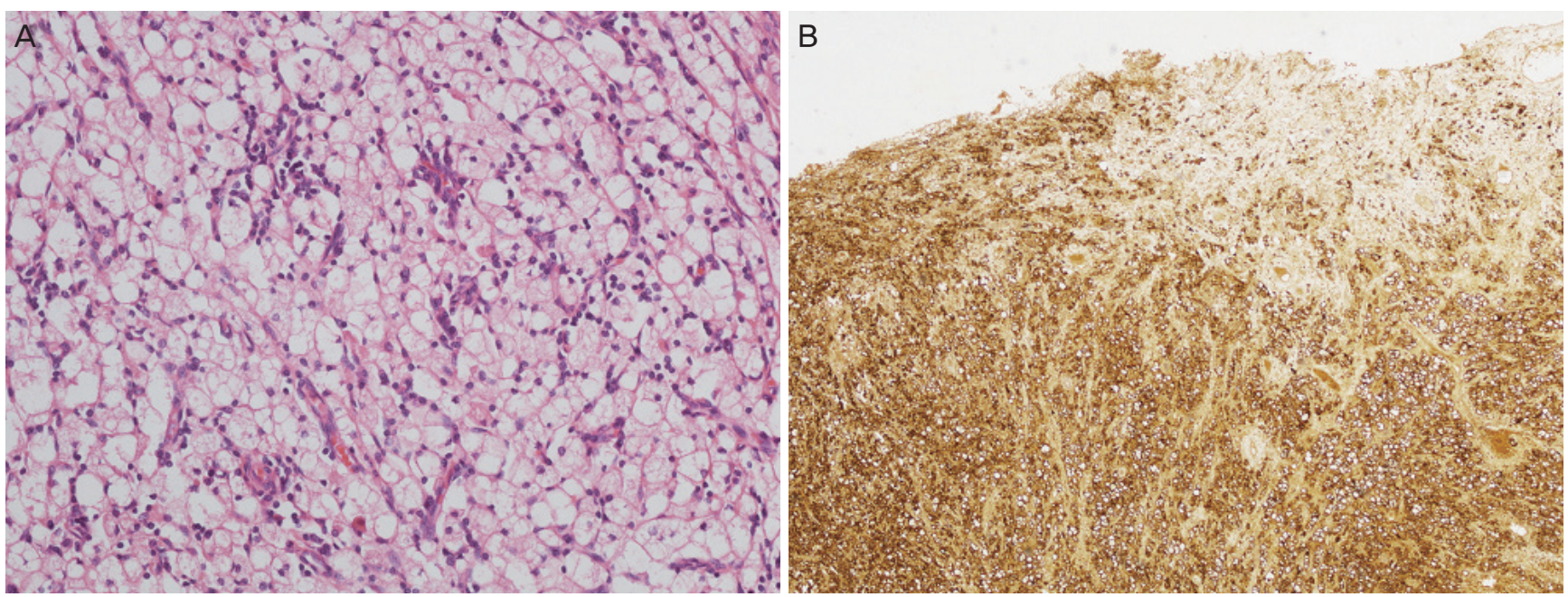

Fig. 2. (A) Image of histopathologic features of the uterus demonstrating clear and foamy histiocytes (hematoxylin and eosin stain, $\times 200$ ). (B) Immunohistochemical staining image demonstrating the strong positive reactivity of histiocytic markers $(C D 68, \times 40)$. 


\section{Obstetrics \& Gynecology Science}

Vol. 63, No. 4, 2020

no atypia or mitosis (Fig. 2A). For differential diagnosis of histiocytic proliferation in the female genital tract, further diagnostic workup including immunohistochemistry (CD68, S100, and cytokeratin) and molecular pathology (BRAF V600E gene) was performed. Immunohistochemical staining was positive for CD68 (Fig. 2B) and negative for S100 and cytokeratin. BRAF V600E gene mutation was absent in realtime PCR. The bone scan revealed no uptake in long bones.

The patient was regularly followed up at 3-month intervals with no medical therapy, and she remained without evidence of recurrence 8 months after surgery.

\section{Discussion}

ECD is an extremely rare type of non-Langerhans cell histiocytosis involving multiple organs including the bones, central nervous system, heart, lung, retroperitoneum, and skin. Skeletal involvement is the most frequent manifestation, with $96 \%$ of patients displaying the characteristic radiological finding of bilateral symmetric long bone sclerosis [4]. However, ECD has heterogeneous and diverse clinical manifestations. Atypical presentations can be challenging for the diagnosis of ECD. ECD with no skeletal involvement is extremely rare, and only a few cases have been reported [5]. Our patient had no skeletal involvement.

Histologically, there is proliferation of foamy lipid-laden histiocytes surrounded by Touton giant cells. Due to the lack of cytological atypia in the cells, the neoplastic nature of the proliferation is often missed. Immunohistochemical staining for CD68, CD163, and FACTOR XIII is positive, with CD1a, Langerin, and $\mathrm{S} 100$ being negative. The BRAF V600E mutation is present in more than $50 \%$ of ECD cases, with other mutations such as N/KRAS and PIK3CA described in BRAFnegative ECD [6].

In a prospective investigation of 60 adults with ECD conducted at the National Institutes of Health $(\mathrm{NIH})$ clinical center, one-third of patients had a history of renal impairment before their $\mathrm{NIH}$ visit [7]. A total of $65 \%$ of patients exhibited encasement of the kidney and involvement of the retroperitoneal space, causing renal artery stenosis in $54 \%$ of cases and ureteropelvic junction obstruction in $51 \%$ of cases [7]. However, a study investigating abdominal involvement during ECD reported that there was no uterine involvement [8].

ECD has no standard therapy, although consensus guide- lines for clinical management were recently reported [9]. Empiric treatments include anti-inflammatory, immunosuppressive, and chemotherapeutic agents, and recent reports of BRAF V600E and MAPK pathway gene mutations in ECD have suggested treatment with BRAK and MEK inhibitors [7].

Our patient had multiple retroperitoneal organ involvement without skeletal bone involvement. Tissue biopsy and immunohistochemical studies suggested ECD diagnosis. However, the BRAF V600E gene mutation was not found. To the best of our knowledge, uterine involvement in ECD has not been reported. Differential diagnosis of ECD in the female genital tract includes a variety of infectious, inflammatory, and neoplastic disorders. Xanthogranulomatous inflammation involving the uterus is typically centered in the endometrium and involves a combination of neutrophils, plasma cells, lymphocytes, and hemosiderin-laden histiocytes [10]. Unlike ECD, uterine Rosai-Dorfman disease involves lymphocytophagocytosis or emperipolesis [11].

Our patient presented atypical ECD with pathologic and immunohistochemical characteristics that coincided with ECD and clinical characteristics such as skeletal bone involvement and BRAF V600E gene mutation that did not coincide with ECD. To the best of our knowledge, this is the first case report of retroperitoneal ECD with mainly uterine involvement. To correctly diagnose atypical ECD, communication among clinicians, radiologists, and pathologists is vital.

\section{Conflict of interest}

No potential conflict of interest relevant to this article was reported.

\section{Ethical approval}

The study was performed in accordance with the principles of the Declaration of Helsinki. Written informed consents were obtained.

\section{Patient consent}

The patients provided written informed consent for the publication and the use of their images. 


\section{Obstetrics \& Gynecology Science}

Hae Min Kim, et al. Retroperitoneal histiocytosis

\section{References}

1. Favara BE, Feller AC, Pauli M, Jaffe ES, Weiss LM, Arico $M$, et al. Contemporary classification of histiocytic disorders. Med Pediatr Oncol 1997;29:157-66.

2. Campochiaro C, Tomelleri A, Cavalli G, Berti A, Dagna L. Erdheim-Chester disease. Eur J Intern Med 2015;26:2239.

3. Haroche J, Arnaud L, Amoura Z. Erdheim-Chester disease. Curr Opin Rheumatol 2012;24:53-9.

4. Mazor RD, Manevich-Mazor M, Shoenfeld Y. ErdheimChester disease: a comprehensive review of the literature. Orphanet J Rare Dis 2013;8:137.

5. Salama H, Kojan S, Abdulrahman S, Azzumeea F, Alhejazi A. Erdheim-Chester disease with no skeletal bone involvement and massive weight loss. Case Rep Hematol 2017;2017:3862052.

6. Emile JF, Diamond EL, Hélias-Rodzewicz Z, Cohen-Aubart F, Charlotte F, Hyman DM, et al. Recurrent RAS and PIK3CA mutations in Erdheim-Chester disease. Blood
2014;124:3016-9.

7. Estrada-Veras JI, O'Brien KJ, Boyd LC, Dave RH, Durham $B, X i L$, et al. The clinical spectrum of Erdheim-Chester disease: an observational cohort study. Blood Adv 2017;1:357-66.

8. Nikpanah M, Kim L, Mirmomen SM, Symons R, Papageorgiou I, Gahl WA, et al. Abdominal involvement in Erdheim-Chester disease (ECD): MRI and CT imaging findings and their association with $\mathrm{BRAF}^{\mathrm{V} 600 \mathrm{E}}$ mutation. Eur Radiol 2018;28:3751-9.

9. Diamond EL, Dagna L, Hyman DM, Cavalli G, Janku F, Estrada-Veras J, et al. Consensus guidelines for the diagnosis and clinical management of Erdheim-Chester disease. Blood 2014;124:483-92.

10. Blanco C, Fernández F, Buelta L, Garijo F, Val-Bernal JF, Sánchez S. Xanthomatous endometritis. Appl Pathol 1989;7:273-6.

11. Pan LY, Offman SL, Warnke RA, Longacre TA. Uterine Rosai-Dorfman disease (sinus histiocytosis with massive lymphadenopathy). Int J Gynecol Pathol 2014;33:432-6. 